\title{
Sigmoidal Transformations and the Trapezoidal Rule
}

\author{
David Elliott*
}

(Received 17 February, 1998)

\begin{abstract}
A sigmoidal transformation is a one-to-one mapping of the compact interval $[0,1]$ onto itself whose graph is $S$-shaped. After giving a formal definition, various mappings already given in the literature are reviewed in the light of the definition. At least one new transformation is introduced and criteria given for generating transformations having special properties. The use of these transformations in using the trapezoidal rule to evaluate $\int_{0}^{1} f(x) d x$
\end{abstract}

*Dept of Mathematics, University of Tasmania, GPO Box 252-37, Hobart, Tasmania 7001, Australia. mailto:elliott@hilbert.maths.utas.edu.au

See http://jamsb.austms.org.au/V40/E006/home.html for this paper and ancillary services, (c) Austral. Mathematical Soc. 1998. Published 12 November 1998, last corrected November 23, 1998. 
is then considered and asymptotic estimates of the truncation errors are obtained under different conditions. The paper concludes with some numerical examples.

\section{Contents}

1 Introduction

E78

2 Examples of "algebraic" sigmoidal transformations

E85

3 Examples of "integral" sigmoidal transformations

E94

4 The Offset Trapezoidal Rule

E107

5 Some numerical results

E127

6 Conclusion

E132

References

E135

\section{Introduction}

We shall start by giving a formal definition of what we mean by a sigmoidal transformation (see Definition 1.1). We shall then show how all the sigmoidal transfor- 
mations we shall consider in this paper are generated from a simple formula (see equation (1)) involving a suitably chosen function $f$. After stating conditions to be satisfied by $f$ we consider in some detail many of the sigmoidal transformations already to be found in the literature. In $\S 2$ we shall consider what I have called "algebraic" transformations of which that due to Kress [8] is an example. These, in turn, lead on to transformations involving exponential functions and associated with the names of Sag \& Szekeres [14], Mori [11], Jeon [6]. In $\S 3$ we consider "integral" transformations which are associated with the names of Korobev [7], Iri, Moriguti \& Takasawa [5], Laurie [9] and Sidi [15]. In each of $\S 2$ and $\S 3$ we introduce a new transformation and consider its properties. All these transformations are associated with the evaluation of integrals of the form $\int_{0}^{1} f(x) d x$ using the general offset trapezoidal rule.

It is well known that the truncation error is given by the Euler-Maclaurin expansion which involves a knowledge of the integrand and its derivatives at the end-points 0 and 1 . As we shall see, a suitably chosen sigmoidal transformation of the variable of integration will allow, in general, an arbitrary number of these derivatives to be zero thereby improving the rate of convergence of the quadrature sum to the integral. For some well known sigmoidal transformations, all the derivatives will be zero and we shall then have a rate of convergence which is exponential.

Our aim, in Section $\S 4$, is to obtain asymptotic forms for the truncation error and this we have been able to do making use of the well known Abel-Plana formula which, however, does require that the definition of $f$ can be extended from $[0,1]$ into a strip $S$ of the complex plane based on $[0,1]$; see (74). For many exam- 
ples this is not a limitation. Two asymptotic estimates are given. In the first, see $\S 4.2$, we assume that $f$ is holomorphic on $[0,1]$; in the second, see $\S 4.3$, we shall assume that $f$ is holomorphic at all points of $(0,1)$ and has algebraic singularities at the points 0 and 1 . In $\S 5$ we give the results of some numerical experiments.

Let us now consider the definition of a sigmoidal transformation and, in particular, a sigmoidal transformation of order $r$.

Definition 1.1 (a) A real-valued function $\gamma$ is said to be a sigmoidal transformation if the following conditions are satisfied:

(i) $\gamma \in C^{1}[0,1] \cap C^{\infty}(0,1)$ with $\gamma(0)=0$;

(ii) $\gamma(x)+\gamma(1-x)=1,0 \leq x \leq 1$;

(iii) $\gamma$ is strictly increasing on $[0,1]$;

(iv) $\gamma^{\prime}$ is strictly increasing on $[0,1 / 2]$ with $\gamma^{\prime}(0)=0$.

(b) If, in addition to (a), either

(i) $\gamma^{(j)}(x)=O\left(x^{r-j}\right)$ near $x=0$, for all $j \in \mathbf{N}_{0}$ where $\mathbf{N}_{0}=\{0,1,2, \ldots\}$ and $r \geq 1$, then $\gamma$ is said to be a sigmoidal transformation of order $r$; or

(ii) $\gamma^{(j)}(0)=0$ for all $j \in \mathbf{N}_{0}$, then $\gamma$ is said to be a sigmoidal transformation of infinite order. 
Examples of these transformations will be given in $\S 2$ and $\S 3$ but let us first consider some general properties arising from this definition.

1. From (a)(i) and (a)(ii) we have that $\gamma(1)=1$. From (a)(iii) we see that $\gamma$ is a one-to-one mapping of the compact interval $[0,1]$ onto itself.

2. From (a)(i), (a)(ii) and (a)(iv) we have that $\gamma^{\prime}(x)=\gamma^{\prime}(1-x)$ so that $\gamma^{\prime}(1)=0$, also. The function $\gamma^{\prime}$ is symmetric about the line $x=1 / 2$. Since, from (a)(iv), $\gamma^{\prime}$ is strictly increasing on $[0,1 / 2]$, it will be strictly decreasing on $[1 / 2,1]$. Thus the function $\gamma^{\prime}$ is "bell-shaped" and has a global maximum at $x=1 / 2$. Again, from (a)(i) and (a)(ii), we have that $\gamma^{\prime \prime}(x)+\gamma^{\prime \prime}(1-x)=0$ on $(0,1)$ so that, in particular, $\gamma^{\prime \prime}(1 / 2)=0$ as one would expect. Because the graph of $\gamma$ is like an elongated $S$ we have elected to call such transformations, sigmoidal transformations (see the New Collins Concise English Dictionary, 1982). They are also known as periodizing transformations (see Laurie [9]).

3. From (a)(ii) and (b)(i) we see that if $\gamma$ is a sigmoidal transformation of order $r$ then, near $x=1$, for all $j \in \mathbf{N}_{0}$,

$$
\gamma^{(j)}(x)=\delta_{0, j}+O\left((1-x)^{r-j}\right),
$$

where $\delta_{0, j}$ is Kronecker's delta. Again, from (a)(ii) and (b)(ii), if $\gamma$ is a sigmoidal transformation of infinite order then although $\gamma(1)=1$, nevertheless we have $\gamma^{(j)}(1)=0$ for all $j \in \mathbf{N}$, where $\mathbf{N}:=\{1,2,3, \ldots\}$. 
We need a way of generating sigmoidal transformations and, from an inspection of the particular sigmoidal transformations to be found in the literature (see $\S \S 2,3)$, the following theorem appears to be particularly useful.

Theorem 1.2 Suppose a real-valued function $f$, defined on $[0,1]$, has the following properties:

(i) $f \in C^{1}[0,1] \cap C^{\infty}(0,1)$ with $f(0)=f^{\prime}(0)=0$ and $f(x)>0$ for

$0<x \leq 1$;

(ii) $\frac{f^{\prime}(x)}{f(x)}+\frac{f^{\prime}(1-x)}{f(1-x)}>0$ for $0<x<1$;

(iii) $\frac{f^{\prime \prime}(x)}{f(x)}-\frac{f^{\prime \prime}(1-x)}{f(1-x)}>2\left(\frac{f^{\prime}(x)-f^{\prime}(1-x)}{f(x)+f(1-x)}\right)\left(\frac{f^{\prime}(x)}{f(x)}+\frac{f^{\prime}(1-x)}{f(1-x)}\right)$ for $0<x<1 / 2$.

Then the function $\gamma$, defined on $[0,1]$ by

$$
\gamma(x)=f(x) /(f(x)+f(1-x)),
$$

is sigmoidal.

Proof. From (i), since $f(x)>0$ for $0<x \leq 1$ then $f(x)+f(1-x)>0$ for $0 \leq x \leq 1$ so that $\gamma$ is defined on $[0,1]$. For all $x \in[0,1]$ we see from (1) that $\gamma(x)+\gamma(1-x)=1$ so that, in particular, since $f(0)=0$ we have $\gamma(0)=0$ and 
$\gamma(1)=1$. From the conditions placed on $f$ in (i) we see that conditions (a)(i) and (ii) of Definition 1.1 are satisfied.

From (1) we have

$$
\gamma^{\prime}(x)=\left(f^{\prime}(x) f(1-x)+f^{\prime}(1-x) f(x)\right) /(f(x)+f(1-x))^{2} .
$$

From (i) and (ii), the numerator is positive on $(0,1)$ and since the denominator is strictly positive we have that $\gamma^{\prime}>0$ on $(0,1)$. Hence $\gamma$ is strictly increasing on $[0,1]$ so that condition (a)(iii) of Definition 1.1 is satisfied.

To prove (a)(iv) of Definition 1.1 we need to show that $\gamma^{\prime \prime}>0$ on $(0,1 / 2)$. On differentiating (2) with respect to $x$ we find, after a little algebra, that condition (iii) implies that $\gamma^{\prime \prime}$ is positive on $(0,1 / 2)$. Hence $\gamma^{\prime}$ is strictly increasing on $[0,1 / 2]$ and, again from $(2), \gamma^{\prime}(0)=0$.

Hence $\gamma$, as defined by equation (1), is a sigmoidal transformation.

Corollary 1.3 Suppose $f$ satisfies conditions (i) to (iii) of Theorem 1.2 and $\gamma$ is defined by equation (1).

(a) If, for all $j \in \mathbf{N}_{0}, f^{(j)}(x)=O\left(x^{r-j}\right)$ near $x=0, r \geq 1$, then $\gamma$ is a sigmoidal transformation of order $r$;

(b) If $f$ and all its derivatives vanish at $x=0$ then $\gamma$ is a sigmoidal transformation of infinite order. 
Proof. The proofs of both parts (a) and (b) follow at once from the definition of $\gamma$ as given in equation (1).

As we shall see below, for a given function $f$, it is sometimes difficult to check out all the conditions of Theorem 1.2, in particular condition (iii) can be troublesome. However, if we use Theorem 1.2 to define $\gamma$ from a given $f$ then we know that $\gamma^{\prime}$ should have a local maximum at $x=1 / 2$.

Theorem 1.4 Suppose $f$ satisfies condition (i) of Theorem 1.2. If

$$
3 f^{\prime}(1 / 2) f^{\prime \prime}(1 / 2)-f(1 / 2) f^{\prime \prime \prime}(1 / 2)>0,
$$

then $\gamma$, as defined by equation (1), satisfies conditions (a)(i) and (a)(ii) of Definition 1.1 and $\gamma^{\prime}$ has a local maximum at $x=1 / 2$.

Proof. The first part has already been proved in Theorem 1.2. On differentiating equation (2) twice with respect to $x$ then we find, after some tedious algebra, that $\gamma^{\prime \prime \prime}(1 / 2)<0$ gives inequality (3).

In the next section we shall consider some of the existing sigmoidal transformations in the light of the analysis given in this section and we shall introduce some new transformations. 


\section{Examples of "algebraic" sigmoidal transformations}

Many sigmoidal transformation, although not called by that name, have already appeared in the literature and we shall look at them again following the discussion of $\S 1$. In addition we shall consider two new transformations and also some of the connections between them.

Transformation 2.1 Perhaps the algebraically simplest and most widely used of these transformations is that obtained by choosing

$$
f(x)=x^{r}, \quad r>1 .
$$

This has been used, for example, by Prössdorf and Rathsfeld [13], Elliott and Prössdorf [2] and Duduchava, Elliott and Wendland [1].

It is immediately obvious that $f$ satisfies conditions (i) and (ii) of Theorem 1.2. For condition (iii) we require

$$
\frac{(r-1)(1-2 x)}{x(1-x)}>-2 r\left(\frac{(1-x)^{r-1}-x^{r-1}}{x^{r}+(1-x)^{r}}\right) \text { on }(0,1 / 2) .
$$

Since $r>1$ and $0<x<1 / 2$ we see that the left hand side of (5) is always positive whereas the right hand side is negative. Thus condition (iii) of Theorem 1.2 is satisfied so that

$$
\gamma_{r}(x):=x^{r} /\left(x^{r}+(1-x)^{r}\right)
$$


is a sigmoidal transformation and, from Corollary 1.3, it is obvious that it is of order $r$. From equation (6) we have that

$$
\ell:=\gamma_{r}^{\prime}(1 / 2)=r,
$$

which obviously increases with $r$. It is readily verified from equation (6) that, for any positive real numbers $p$ and $r$, this transformation has the beautiful property

$$
\gamma_{p}\left(\gamma_{r}(x)\right)=\gamma_{r}\left(\gamma_{p}(x)\right)=\gamma_{p r}(x) .
$$

From this it immediately follows that the inverse of $\gamma_{r}$ is $\gamma_{1 / r}$, although for $r>1$ it is obvious that $\gamma_{1 / r}$ is not a sigmoidal transformation according to Definition 1.1.

Transformation 2.2 We shall now look at a variant of Transformation 2.1 which has the virtue that $\ell=\gamma_{r}^{\prime}(1 / 2)$ is independent of $r$. We shall write

$$
f(x)=\left(x-c E_{2}(x)\right)^{r}, \quad r>1,
$$

where $c$ is a constant to be determined and $E_{2}$ denotes the Euler polynomial of degree 2 defined by

$$
E_{2}(x)=x^{2}-x,
$$

(see, for example Gradshteyn \& Ryzhik $[4, \S 9.6])$. Now

$$
f(x)=(1+c)^{r} x^{r}(1-(c /(1+c)) x)^{r}
$$


so that if $\gamma$ is to be of order $r$ we must have $1+c>0$ or $c>-1$. In this case condition (i) of Theorem 1.2 is satisfied. Condition (ii) of Theorem 1.2 requires that

$$
1+\frac{c}{2}+2 c\left(x-\frac{1}{2}\right)^{2}>0
$$

on $(0,1)$ and again this is true if $c>-1$.

Since $\ell:=\gamma^{\prime}(1 / 2)$ we find, with $f$ defined by equation (9), that

$$
c=2(r / \ell-1) \text {. }
$$

Now $c>-1$ implies that $r>\ell / 2$ and since we have already assumed $r>1$ we shall henceforth assume that

$$
r>\max (1, \ell / 2) .
$$

Condition (iii) of Theorem 1.2 appears intractable in this case and if we replace it by the weaker condition given by inequality (3) we find, after some algebra, that we must have

$$
\left(\ell^{2}-3\right) r^{2}+3 \ell r-\ell^{2}>0 .
$$

This will certainly be satisfied if we assume

$$
\ell>\sqrt{3} \text { and } r>\max (1, \ell / 2) .
$$

Thus choosing $\ell$ and $r$ satisfying (15), with $c$ given by equation (12) and $f$ defined by equation (9), we claim that the $\gamma$ defined by equation (1) will be a sigmoidal transformation. In particular, with $\ell=2$ and $r>1, c=r-2$ will given a sigmoidal transformation $\gamma_{r}$ or order $r$ such that $\gamma_{r}^{\prime}(1 / 2)=2$. It is of interest to investigate what happens to this transformation as $r \rightarrow \infty$. 
Transformation 2.3 From Transformation 2.2 we can write, from equation (1),

$$
\gamma_{r}(x)=1 /\left(1+F_{r}(x)\right),
$$

where

$$
\begin{aligned}
F_{r}(x) & =f(1-x) / f(x) \\
& =\frac{\left(1+\frac{\ell}{r}\left(\frac{1}{2 x}-1\right)\right)^{r}}{\left(1-\frac{\ell}{r}\left(1-\frac{1}{2(1-x)}\right)\right)^{r}},
\end{aligned}
$$

on using equations (9), (10) and (12). On letting $r \rightarrow \infty$ we obtain a limit function $F_{\infty}$ say, where

$$
F_{\infty}(x)=\exp \left[\frac{\ell}{2}\left(\frac{1}{x}-\frac{1}{1-x}\right)\right] .
$$

This gives rise to a sigmoidal transformation, which we shall denote by $\gamma_{\infty}^{S S}$, defined by

$$
\gamma_{\infty}^{S S}(x)=\frac{1}{1+F_{\infty}(x)}=\frac{1}{2}+\frac{1}{2} \tanh \left[\frac{\ell}{4}\left(-\frac{1}{x}+\frac{1}{1-x}\right)\right] .
$$

This transformation turns out to be the one introduced by Sag and Szekeres [14] in the context of evaluating multi-dimensional integrals over the unit hypercube. We might note in passing that $\gamma_{\infty}^{S S}$ can be obtained by choosing either

$$
f(x)=\exp \left(-\frac{\ell}{2 x}\right) \text { or } f(x)=\exp \left[\frac{\ell}{4}\left(-\frac{1}{x}+\frac{1}{1-x}\right)\right]
$$


Using the first of these representations it is readily shown that $f$ satisfies conditions (i) and (ii) of Theorem 1.2 for any $\ell>0$ but the weaker inequality (3) requires, not surprisingly, that $\ell>\sqrt{3}$ in order that $\gamma_{\infty}^{S S}$ have a local maximum at $1 / 2$.

At this point we have the choice of two directions open to us. Either we continue with sigmoidal transformations of infinite order (and there are quite a few of these) or we return to the spirit of Transformation 2.2 where we look for sigmoidal transformations of order $r$ with a bounded value of $\gamma^{\prime}(1 / 2)$. We consider the latter first.

Transformation 2.4 This transformation was first considered by Kress [8]. Transforming what he has defined over the interval $[0,2 \pi]$ to the interval $[0,1]$ we choose

$$
f(x)=\left(x+c B_{3}(x)\right)^{r}
$$

where $r>1, c$ is a constant to be determined and $B_{3}$ is the Bernoulli polynomial of degree 3 defined by

$$
B_{3}(x):=x(x-1 / 2)(x-1),
$$

see Gradshteyn \& Ryzhik [4, §9.62].

Now since we want $\gamma$ to be of order $r$ we have, near $x=0$, that

$$
f(x)=(1+c / 2)^{r} x^{r}(1+O(x))
$$


so that we must have $c>-2$. Again, from condition (ii) of Theorem 1.2 we find that this reduces to requiring

$$
1+c B_{3}^{\prime}(x)>0 \text { on }(0,1)
$$

which in turn requires $c<4$. Thus we have $-2<c<4$. But again, with $\ell=\gamma^{\prime}(1 / 2)$ we find that

$$
c=4(1-\ell / r)
$$

and, since $-2<c<4$, we must have

$$
0<\ell / r<3 / 2 .
$$

Since $\ell>0$ and $r>1$ the left hand inequality is readily satisfied but the right hand inequality requires that $r>2 \ell / 3$. Again condition (iii) of Theorem 1.2 is too difficult to apply directly but the weaker condition (3) implies, after a little algebra, that

$$
\left(r^{2}-1\right)(\ell / r)^{3}>3(1-\ell / r) .
$$

This inequality essentially gives us an upper limit on $r$ for a given value of $\ell$. In Table 2.1 we have recorded for $\ell=2(0.5) 4$ the maximum value of $r$ allowed by equation (23). 


\begin{tabular}{lcr}
\multicolumn{1}{c}{$\ell$} & $r_{\min }=2 \ell / 3$ & \multicolumn{1}{c}{$r_{\max }$} \\
\hline \hline 2 & 1.333 & 4.537 \\
2.5 & 1.667 & 7.619 \\
3 & 2.000 & 11.937 \\
3.5 & 2.333 & 17.746 \\
4 & 2.667 & 25.300
\end{tabular}

Table 2.1: Range of $r$ for a given $\ell$

Thus our analysis indicates that for a given value of $\ell$ if we choose $r$ in the interval $\left(r_{\min }, r_{\max }\right)$ then we expect the transformation defined by equations (1), (19) and (21) to be sigmoidal of order $r$.

There are a few transformations to be found in the literature which are of a similar nature to that given by Sag and Szekeres. We consider briefly just two of them.

Transformation 2.5 Mori [11] has given a transformation mapping $[-1,1]$ onto itself. Transforming this to a mapping of $[0,1]$ onto itself and using the notation of this paper, Mori's transformation corresponds to choosing

$$
f(x)=\exp \left[\ell \sinh \left(\frac{1}{4(1-x)}-\frac{1}{4 x}\right)\right]
$$


so that the transformation $\gamma_{\infty}^{M}$ say, is of infinite order and is given by

$$
\gamma_{\infty}^{M}(x)=\frac{1}{2}+\frac{1}{2} \tanh \left[\ell \sinh \left(\frac{1}{4(1-x)}-\frac{1}{4 x}\right)\right],
$$

which should be compared with equation (17). Again, we have $\gamma_{\infty}^{M}(1 / 2)=\ell$. From inequality (3), the requirement that $\gamma_{\infty}^{M^{\prime}}$ has a local maximum at $1 / 2$ gives, after some tedious algebra, that $\ell>\sqrt{7 / 2}=1.870829$ to $6 \mathrm{D}$.

Transformation 2.6 Jeon [6] has introduced a transformation which has overtones of both the Sag and Szekeres and Mori transformations as well as the Kress transformation.

If $\alpha, \beta>0$ and if $p$ is defined by

$$
p=\alpha 2^{\beta}(1+\beta \log 2),
$$

then, with

$$
v(x)=x+(4-8 / p) B_{3}(x),
$$

Jeon's transformation is obtained by choosing the function $f$ to be

$$
f(x)=\exp \left[\alpha(v(x))^{-\beta} \log v(x)\right] .
$$

With this choice of $p$ and for any values of $\alpha$ and $\beta$ we find that Jeon's transformation, $\gamma_{\infty}^{J}$ say, has the property that $\gamma_{\infty}^{J^{\prime}}(1 / 2)=2$. As with Transformations 2.3 
and 2.5 all derivatives of $\gamma_{\infty}^{J}$ of all orders vanish at both 0 and 1 . Now $\gamma_{\infty}^{J}$ is not sigmoidal, according to Definition 1.1, for all values of $\alpha, \beta>0$. In fact requiring the derivative to have a local maximum at $1 / 2$ (see (3)) requires that $\alpha$ and $\beta$ satisfy the inequality

$$
\begin{aligned}
& \alpha^{2}(1+\beta \log 2)^{3}\left(14-3 \alpha \cdot 2^{\beta}(1+\beta \log 2)\right)> \\
& 2^{2-2 \beta}\left[(1+\beta \log 2)\left(\beta^{2}+3 \beta+2\right)+\beta(2 \beta+3)\right] .
\end{aligned}
$$

This appears to be too complicated to take any further but it is readily checked for given values of $\alpha$ and $\beta$.

Jeon's transformation seems to be unnecessarily complicated involving, as it does, two parameters $\alpha$ and $\beta$ and introducing the cubic $v$ in order to have a bounded derivative on $[0,1]$. A similar result in the spirit of Jeon's transformation is obtained by choosing

$$
f(x)=\exp [\ell \cdot \log x /(2(1+\log 2) x)] .
$$

It is readily verified that $\gamma^{\prime}(1 / 2)=\ell$. Obviously, all derivatives of the corresponding $\gamma$ vanish at both zero and one and we have $\gamma(0)=0, \gamma(1)=1$. On applying inequality (3) we find that $\gamma$ has a local maximum at $x=1 / 2$ provided that

$$
\ell>((11+6 \log 2) /(2+2 \log 2))^{1 / 2}=2.115874,
$$

to $6 \mathrm{D}$. We do not propose to proceed any further in this paper with this transformation; it is merely noted in passing. 


\section{Examples of "integral" sigmoidal transformations}

We now consider transformations in which the function $f$ is given as the integral of some function $h$. To be more explicit, let

$$
f(x)=\int_{0}^{x} h(\xi) d \xi .
$$

If the function $h$ satisfies the condition

$$
h(\xi)=h(1-\xi), \quad 0 \leq \xi \leq 1,
$$

then we have simply from equation (1) that

$$
\gamma(x)=(1 / Q) \int_{0}^{x} h(\xi) d \xi, \quad 0 \leq x \leq 1,
$$

where

$$
Q:=\int_{0}^{1} h(\xi) d \xi .
$$

In other words, from equations (32) and (33) we have that

$$
f(x)+f(1-x)=Q,
$$

a constant. We now need conditions on $h$ for $\gamma$ to be sigmoidal. 
(i) $h \in C[0,1] \cap C^{\infty}(0,1)$ with $h(0)=0$ and $h(x)>0$ for $0<x \leq 1$,

(ii) $h(x)=h(1-x), \quad 0 \leq x \leq 1$,

(iii) $h^{\prime}(x)>0$ on $(0,1 / 2]$,

(iv) $h^{(j)}(x)=O\left(x^{r-1-j}\right)$ near $x=0$, for all $j \in \mathbf{N}_{0}$.

Then $\gamma$, as defined by equations (34) and (35), is a sigmoidal transformation of order $r$.

Proof. We check this against the requirements of Definition 1.1. From (i) and (ii) we have that

$$
f(x)+f(1-x)=\int_{0}^{1} h(\xi) d \xi=Q>0 .
$$

Thus $\gamma$ is defined on $[0,1]$ and furthermore, from (ii) again, we have that $\gamma(x)+$ $\gamma(1-x)=1$ for $0 \leq x \leq 1$. Now $\gamma^{\prime}(x)=h(x) / Q$ which is positive on $(0,1)$ so that $\gamma$ is increasing on $[0,1]$. Furthermore, $\gamma^{\prime}(0)=0$. Now $\gamma^{\prime \prime}(x)=h^{\prime}(x) / Q$ and from (iii) we have that $\gamma^{\prime}$ is strictly increasing on $[0,1 / 2]$. Thus conditions (a)(i) to (a)(iv) of Definition 1.1 are satisfied. Finally as a consequence of (iv) and from the definition of $\gamma$ we have that $\gamma^{(j)}(x)=O\left(x^{r-j}\right)$ near $x=0$, for all $j \in \mathbf{N}_{0}$, so that $\gamma$ is a sigmoidal transformation of order $r$. 
We note that the conditions of this theorem are considerably simpler than those of Theorem 1.2 which we used in the previous section for "algebraic" sigmoidal transformations. Let us now consider some examples.

Transformation 3.2 Korobev [7] chooses

$$
h(x)=(x(1-x))^{r-1} \quad \text { with } r>1 .
$$

It is readily verified that this $h$ satisfies conditions (i)-(iv) of Theorem 3.1. Now

$$
Q=\int_{0}^{1}(x(1-x))^{r-1} d x=(\Gamma(r))^{2} / \Gamma(2 r),
$$

see Gradshteyn and Ryzhik [4, §3.191(3)].

For $r \gg 1$ we have that $Q \sim \pi^{1 / 2} /\left(2^{2 r-1} r^{1 / 2}\right)$. Consequently

$$
\gamma^{\prime}(1 / 2) \sim\left(2 / \pi^{1 / 2}\right) r^{1 / 2} \quad \text { for } r \gg 1,
$$

which increases as $r$ increases.

Transformation 3.3 In the spirit of the Sag, Szekeres transformation (see Transformation 2.3) Iri, Moriguti and Takasawa [5] have chosen

$$
h(x)=\exp [-(1 / x+1 /(1-x))]
$$


Again, it is readily verified that $h$ satisfies conditions (i)-(iii) of Theorem 3.1 so that $\gamma$ as defined in (34) is a sigmoidal transformation. In this case it is obvious that $\gamma^{(k)}$ vanishes at both 0 and 1 for all $k \in \mathbf{N}$ so that this is a sigmoidal transformation of infinite order.

In this case $Q=0.00702986$ to 6 significant digits (see [5, equation (1.3)]) and we find that $\gamma^{\prime}(1 / 2)=2.605406$, correct to 6 decimal places.

Transformation 3.4 An important transformation has been given by Sidi [15] who chooses

$$
h(x)=(\sin (\pi x))^{r-1}, \quad \mathbf{N} \ni r \geq 2 .
$$

Again we see that $h$ satisfies conditions (i) to (iv) of Theorem 3.1 so that the corresponding transformation $\gamma_{r}$, is sigmoidal and of order $r$. We have, see Gradshteyn and Ryzhik [4, §3.621], that

$$
Q=\int_{0}^{1}(\sin (\pi x))^{r-1} d x=\Gamma(r / 2) /\left(\pi^{1 / 2} \Gamma(r / 2+1 / 2)\right)
$$

so that, in particular,

$$
\gamma_{r}^{\prime}(1 / 2) \sim(\pi r / 2)^{1 / 2} \quad \text { for } r \gg 1,
$$

which is unbounded as $r$ increases.

On integration by parts we find, after a little algebra, that for $r \geq 1, \gamma_{r}$ satisfies the recurrence relation

$$
\gamma_{r+1}(x)=\gamma_{r-1}(x)-\frac{\Gamma(r / 2)}{2 \pi^{1 / 2} \Gamma(r / 2+1 / 2)}(\sin (\pi x))^{r-1} \cos (\pi x),
$$


if we define $\gamma_{0}(x):=1 / 2$ and $\gamma_{1}:=x$. We have, in particular, that

$$
\begin{aligned}
& \gamma_{2}(x)=(1-\cos (\pi x)) / 2, \quad \text { and } \\
& \gamma_{3}=x-\frac{1}{2 \pi} \sin (2 \pi x) .
\end{aligned}
$$

More generally, on using the expression for $(\sin (\pi \xi))^{2 m}$ as given in Gradshteyn and Ryzhik $[4, \S 1.320(1)]$ and integrating term by term, we find

$$
\gamma_{2 m+1}(x)=x+2 \sum_{s=1}^{m} \frac{(-1)^{s}(m !)^{2}}{(m-s) !(m+s) !}\left(\frac{1}{2 \pi s}\right) \sin (2 \pi s x),
$$

for all $m \in \mathbf{N}$. We shall consider the importance of this particular odd order sigmoidal transformation later when we consider the Euler-Maclaurin summation formula (see $\S 4.2$ ).

Transformation 3.5 Laurie [9] looks for an integral sigmoidal transformation of order $r$ where $r$ is an odd integer greater than or equal to 3 such that the function $h$, in addition to satisfying (33), also satisfies

$$
\left\{\begin{array}{l}
h^{(2 i)}(0)=0 \text { for } i=0(1)((r-3) / 2), \\
h^{(2 i-1)}(0)=0 \text { for } i=1(1)(r-1)
\end{array}\right.
$$


We see that (33) and (45) together imply that $h$ also satisfies the same homogeneous conditions as given in (45) but at the end-point 1 . Thus there is a total of $3(r-1)$ homogeneous conditions to be satisfied and Laurie looks for a polynomial solution of degree $3(r-1)$. To this end recall that the Bernoulli polynomials $B_{2 j}, j \in \mathbf{N}_{0}$, of even order satisfy

$$
B_{2 j}(x)=B_{2 j}(1-x) .
$$

(see, for example, Gradshteyn \& Ryzhik [4, §9.623(4)]) and write

$$
h(x)=\sum_{j=1}^{3(r-1) / 2} c_{j}\left(B_{2 j}(x)-B_{2 j}\right),
$$

where $c_{3(r-1) / 2}=1$, and $B_{2 j}$ denotes the Bernoulli number which is equal to $B_{2 j}(0)$. This is a polynomial of exact degree $3(r-1)$ and it satisfies $h(x)=$ $h(1-x)$ together with $h(0)=0$. Recall that

$$
B_{2 j}^{(i)}(x)=\frac{(2 j) !}{(2 j-i) !} B_{2 j-i}(x)
$$

so that from the second of (45) we have

$$
0=h^{(2 i-1)}(0)=\sum_{j=1}^{3(r-1) / 2} c_{j} \frac{(2 j) !}{(2 j-2 i+1) !} B_{2 j-2 i+1} .
$$

If we recall that all odd order Bernoulli numbers are zero except for $B_{1}$ which is equal to $-1 / 2$ we see that equation (49) imply that

$$
c_{i}=0 \text { for } i=1(1)(r-1) .
$$


Consequently we may rewrite (47) as

$$
h(x)=\sum_{j=r}^{3(r-1) / 2} c_{j}\left(B_{2 j}(x)-B_{2 j}\right),
$$

with $c_{3(r-1) / 2}=1$. It now remains to satisfy $h^{(2 i)}(0)=0$ for $i=1(1)((r-3) / 2)$ since $h(0)=0$ is satisfied automatically. Again, using (48) we find

$$
\sum_{j=r}^{3(r-1) / 2} c_{j} \frac{(2 j) ! B_{2 j-2 i}}{(2 j-2 i) !}=0 \quad \text { for } i=1(1)((r-3) / 2),
$$

which we can rewrite as

$$
\sum_{j=r}^{(3 r-5) / 2} \frac{(2 j) ! B_{2 j-2 i}}{(2 j-2 i) !} c_{j}=-\frac{(3 r-3) ! B_{3(r-1)-2 i}}{(3(r-1)-2 i) !},
$$

for $i=1(1)((r-3) / 2)$, giving us $(r-3) / 2$ equations for the $(r-3) / 2$ unknowns $c_{r}, c_{r+1}, \ldots, c_{(3 r-5) / 2}$. The author has been unable to determine whether this system of equations has a unique solution for all odd integers $r$. However, Laurie [9] quotes Schneider (private communication) who claims that this is true.

With $h$ given by (51) we find from (34) and (35) that

$$
\gamma_{r}(x)=x-\frac{\sum_{j=r}^{3(r-1) / 2}\left(c_{j} /(2 j+1)\right) B_{2 j+1}(x)}{\sum_{j=r}^{3(r-1) / 2} c_{j} B_{2 j}} .
$$


Let us now consider the three examples discussed by Laurie [9].

(i) $r=3$ From (51) we see immediately that

$$
h(x)=B_{6}(x)-B_{6}
$$

and, from (54),

$$
\begin{aligned}
\gamma_{3}(x) & =x-6 B_{7}(x) \\
& =7 x^{3}-21 x^{5}+21 x^{6}-6 x^{7}
\end{aligned}
$$

which is $O\left(x^{3}\right)$ as expected. Also, $\gamma_{3}^{\prime}(1 / 2)=63 / 32$.

(ii) $\boldsymbol{r}=\mathbf{5}$ From (53) we find $c_{5}=10 / 3$ and with $c_{6}=1$ we have from (54) that

$$
\begin{aligned}
\gamma_{5}(x)= & x+\frac{210}{53}\left(130 B_{11}(x)+33 B_{13}(x)\right) \\
= & \left(3003 x^{5}-17160 x^{7}+85085 x^{9}-150150 x^{10}\right. \\
& \left.+117390 x^{11}-45045 x^{12}+6930 x^{13}\right) / 53 .
\end{aligned}
$$

Again, as expected, $\gamma_{5}(x)=O\left(x^{5}\right)$ and we have

$$
\gamma_{5}^{\prime}(1 / 2)=285,285 / 108,544=2.628289
$$

correct to $6 \mathrm{D}$. 
(iii) $\boldsymbol{r}=7$ From equation (53) we find

$$
c_{7}=\frac{3123}{269}, \quad c_{8}=\frac{10395}{1076} \quad \text { with } c_{9}=1 .
$$

With these values,

$$
\gamma_{7}(x)=x-\frac{56488824}{1145} B_{15}(x)-\frac{8295210}{229} B_{17}(x)-\frac{768264}{229} B_{19}(x)
$$

and it can be verified that

$$
\gamma_{7}(x)=\frac{112404}{229} x^{7}+O\left(x^{9}\right) .
$$

Finally we note that $\gamma_{7}^{\prime}(1 / 2)=23686236 / 7503872=3.156535$, correct to 6D. Thus it appears that $\gamma_{r}^{\prime}(1 / 2)$ is increasing with $r$ but whether $\gamma_{r}^{\prime}(1 / 2)$ is bounded above is not known.

Sidi's Transformation 3.4 has the property that $\gamma_{r}^{\prime}(1 / 2)$ diverges as $r \rightarrow \infty$. We now consider a transformation which is similar to the Sidi odd order transformation but for which $\gamma_{2 m+1}^{\prime}(1 / 2)$ is bounded for all $m \in \mathbf{N}$. In place of equation (32) let us now write

$$
f(x)=\int_{0}^{x}(x-\xi) h(\xi) d \xi
$$

for some $h$. This is essentially a repeated integral of equation (32). We shall assume now that $h$ satisfies the condition

$$
h(\xi)+h(1-\xi)=0, \quad 0 \leq \xi \leq 1 .
$$


Then, from equation (1),

$$
\gamma(x)=\int_{0}^{x}(x-\xi) h(\xi) d \xi / \int_{0}^{1}(x-\xi) h(\xi) d \xi .
$$

The denominator in equation (60) appears at first sight to depend on $x$ but, from equation (59), we see that $\int_{0}^{1} h(\xi) d \xi=0$. Thus we can rewrite equation (60) as

$$
\gamma(x)=\int_{0}^{x}(x-\xi) h(\xi) d \xi / \int_{0}^{1}(1-\xi) h(\xi) d \xi .
$$

With these preliminary comments we need further conditions on $h$ in order that $\gamma$ be a sigmoidal transformation of order $r$.

Theorem 3.6 Suppose $h$ is such that

(i) $h \in C[0,1] \cap C^{\infty}(0,1)$ with $h(x)>0$ for $x \in(0,1 / 2)$,

(ii) $h(x)+h(1-x)=0$ for $0 \leq x \leq 1$,

(iii) $h^{(j)}(x)=O\left(x^{r-2-j}\right)$, near $x=0$, for all $j \in \mathbf{N}_{0}$ and $r>1$.

Then $\gamma$, as defined by equation (61), is a sigmoidal transformation of order $r$. 
Proof. First we observe, on using (i) and (ii), that

$$
\begin{aligned}
\int_{0}^{1}(1-\xi) h(\xi) d \xi & =\int_{0}^{1 / 2}(1-\xi) h(\xi) d \xi+\int_{0}^{1 / 2} \xi h(1-\xi) d \xi \\
& =\int_{0}^{1 / 2}(1-2 \xi) h(\xi) d \xi>0
\end{aligned}
$$

since $1-2 \xi>0$ on $(0,1 / 2)$ and, from (i), $h>0$ on $(0,1 / 2)$. Thus $\gamma$ in equation (61) is defined, with $\gamma(0)=0$ and $\gamma(1)=1$. Again, using (ii), $\gamma(x)+\gamma(1-x)=1$ for $0 \leq x \leq 1$.

From (61),

$$
\gamma^{\prime}(x)=\int_{0}^{x} h(\xi) d \xi / \int_{0}^{1}(1-\xi) h(\xi) d \xi .
$$

We shall now show that $\int_{0}^{x} h(\xi) d \xi>0$ for $0<x<1$. Since $h>0$ on $(0,1 / 2)$ we have that the result is true for $0<x \leq 1 / 2$. For $1 / 2 \leq x<1$ we have, on using (ii),

$$
\begin{aligned}
\int_{0}^{x} h(\xi) d \xi & =\left(\int_{0}^{1}-\int_{x}^{1}\right) h(\xi) d \xi \\
& =\int_{x}^{1} h(1-\xi) d \xi=\int_{0}^{1-x} h(\xi) d \xi>0
\end{aligned}
$$

since $0<1-x \leq 1 / 2$, i.e. $1 / 2 \leq x<1$. Thus $\gamma^{\prime}>0$ on $(0,1)$ and $\gamma$ is strictly increasing on $(0,1)$ with $\gamma(0)=0$ and $\gamma(1)=1$.

Again, $\gamma^{\prime \prime}(x)=h(x) / \int_{0}^{1}(1-\xi) h(\xi) d \xi$ which by (i), is $>0$ on $(0,1 / 2)$ so that $\gamma^{\prime}$ is strictly increasing on $(0,1 / 2)$ with, from equation $(62), \gamma^{\prime}(0)=0$. 
Finally from (i) and (iii) we see that $\gamma(x)=O\left(x^{r}\right)$ near $x=0$ and $\gamma \in$ $C^{1}[0,1] \cap C^{\infty}(0,1)$ so that $\gamma$ is a sigmoidal transformation of order $r$.

Transformation 3.7 Suppose we choose

$$
h(\xi)=\sin ^{2 m-1}(2 \pi \xi), \quad m \in \mathbf{N} .
$$

This function satisfies the conditions of theorem 3.6 to give a sigmoidal transformation

$$
\gamma_{2 m+1}(x)=\int_{0}^{x}(x-\xi) \sin ^{2 m-1}(2 \pi \xi) d \xi / \int_{0}^{1}(1-\xi) \sin ^{2 m-1}(2 \pi \xi) d \xi,
$$

of order $2 m+1$. It is convenient to define

$$
\gamma_{1}(x)=x
$$

and then equation (64) gives, when $m=1$,

$$
\gamma_{3}(x)=x-\frac{1}{2 \pi} \sin (2 \pi x),
$$

which is identical to the third order Sidi transformation, see (43). In fact, judicious integration of equation (64) by parts gives the recurrence relation

$$
\gamma_{2 m+1}(x)=\gamma_{2 m-1}(x)-\frac{\Gamma(m-1 / 2)}{2 \pi^{3 / 2}(2 m-1) \Gamma(m)} \sin ^{2 m-1}(2 \pi x), \quad \text { for } m \in \mathbf{N} .
$$


From Gradshteyn and Ryzhik [4, §3.821(2)] we find

$$
\int_{0}^{1}(1-\xi) \sin ^{2 m-1}(2 \pi \xi) d \xi=\frac{\Gamma(m)}{\left(4 \pi^{1 / 2} \Gamma(m+1 / 2)\right)} .
$$

Then, from equations (64) and (66), we find

$$
\gamma_{2 m+1}^{\prime}(1 / 2)=\frac{4 \pi^{1 / 2} \Gamma(m+1 / 2)}{\Gamma(m)} \int_{0}^{1 / 2} \sin ^{2 m-1}(2 \pi \xi) d \xi .
$$

Again from Gradshteyn and Ryzhik [4, §3.621(1)] we obtain

$$
\gamma_{2 m+1}^{\prime}(1 / 2)=2, \quad \text { for all } m \in \mathbf{N},
$$

which, of course, is independent of $m$.

From equation (64) we may write $\gamma_{2 m+1}$ as a finite sum. Making use of Gradshteyn and Ryzhik $[4, \S 1.320(3)]$ we find, after some algebra, that

$$
\gamma_{2 m+1}(x)=x+\frac{2(\Gamma(m+1 / 2))^{2}}{\pi^{2}} \sum_{s=1}^{m} \frac{(-1)^{s} \sin [2 \pi(2 s-1) x]}{\Gamma(m-s+1) \Gamma(m+s)(2 s-1)^{2}},
$$

for all $m \in \mathbf{N}$.

Finally, let us consider the explicit behaviour of $\gamma_{2 m+1}(x)$ near $x=0$. For $x$ close to 0 we have from equation (64), on replacing $\sin (2 \pi \xi)$ by $(2 \pi \xi)$, that

$$
\gamma_{2 m+1}(x)=\frac{4 \pi^{1 / 2} \Gamma(m+1 / 2)}{\Gamma(m)} \int_{0}^{x}(x-\xi)(2 \pi \xi)^{m-1} d \xi
$$


approximately. Integrating gives

$$
\gamma_{2 m+1}(x)=\frac{2^{2 m-1} \pi^{2 m-1 / 2} \Gamma(m+1 / 2)}{(m+1 / 2) \Gamma(m+1)} x^{2 m+1}+O\left(x^{2 m+3}\right),
$$

for $m \in \mathbf{N}$. We observe that near $x=0$ the expansion of $\gamma_{2 m+1}$ is of the form $x^{2 m+1} \sum_{k=0}^{\infty} a_{k} x^{2 k}$, for some coefficients $a_{k}$. That is, the expansion of $\gamma_{2 m+1}$ near the origin involves only every second power of $x$, an observation which will be important later (see $\S 4.2$ ).

\section{The Offset Trapezoidal Rule}

One of the most important application of sigmoidal transformations is in the approximate evaluation of integrals of the form $\int_{0}^{1} f(x) d x$ by means of the offset trapezoidal rule. The aim of this section is to give asymptotic estimates of the truncation error. In order to do this, we introduce the Abel-Plana formula for the truncation error which, although dating back to the early nineteenth century, proves to be particularly useful in this context. We give asymptotic errors for two distinct cases. In the first, see $\S 4.2$, we assume that $f$ is holomorphic on $[0,1]$; for the second, see $\S 4.3$, we assume that $f$ is holomorphic only on $(0,1)$ with algebraic singularities at the end-points 0 and 1 . Numerical examples of the estimates we obtain are given in $\S 5$. 


\subsection{The Offset Trapezoidal Rule and the Abel-Plana formula}

We use notation introduced by Lyness [10]. Suppose that

$$
t_{\nu}=(\nu+1) / 2 \text { for }-1<\nu \leq 1,
$$

so that $0<t_{\nu} \leq 1$ with, in particular, $t_{0}=1 / 2$ and $t_{1}=1$. The offset trapezoidal rule $Q_{n}^{[\nu]} f, n \in \mathbf{N},-1<\nu \leq 1$ is defined by

$$
Q_{n}^{[\nu]} f:= \begin{cases}\frac{1}{n} \sum_{j=0}^{n-1} f\left(\left(j+t_{\nu}\right) / n\right), & -1<\nu<1, \\ \frac{1}{n} \sum_{j=0}^{n}{ }^{\prime \prime} f(j / n), & \nu=1,\end{cases}
$$

where $\sum^{\prime \prime}$ denotes a sum whose first and last terms are halved. If

$$
\text { If }:=\int_{0}^{1} f(t) d t
$$

then we want an expression for the truncation error $E_{n}^{[\nu]} f$ where

$$
E_{n}^{[\nu]} f:=I f-Q_{n}^{[\nu]} f .
$$

Although we shall assume throughout that $f$ is a real function on $[0,1]$, we shall assume that the definition of $f$ may be continued into the strip $S$ of the complex $z$-plane defined by

$$
S:=\{z: 0 \leq x=\Re z \leq 1, \quad-\infty<y=\Im z<\infty\} .
$$

We can now give the Abel-Plana formula (see, for example, Olver [12]). 
Theorem 4.1 (Abel-Plana). Suppose that

(i) $f$ is continuous in $S$ and holomorphic in $\operatorname{int}(S)$;

(ii) $f(z)=o(\exp (2 \pi n|\Im z|))$ as $\Im z \rightarrow \pm \infty$ in $S$,

uniformly with respect to $\Re z$.

Then

$$
E_{n}^{[\nu]} f=-2 \Im\left\{\int_{0}^{\infty} \frac{f(1+i y)-f(i y)}{\left\{\exp \left[2 \pi\left(n y+i t_{\nu}\right)\right]-1\right\}} d y\right\}
$$

Proof. Let $\mathcal{C}_{1}=A B C D$ be a contour in $S \cap(\Im z \leq 0)$ where $z=-i y, 0 \leq$ $y \leq Y$ along $A B ; z=x-i Y, 0 \leq x \leq 1$ along $B C$; and $z=1-i y, Y \geq y \geq 0$ along $C D$. Similarly in $S \cap(\Im z \geq 0)$ we let $\mathcal{C}_{2}=E F G H$ be the contour where $z=1+i y, 0 \leq y \leq Y$ along $E F ; z=x+i Y, 1 \geq x \geq 0$ along $F G$; and finally $z=i y, Y \geq y \geq 0$ along $G H$.

If $t_{\nu}=1$, then we modify these contours by semicircular indentations in the neighbourhoods of the points 0 and 1 . Since $f$ is holomorphic in $S$ we have, by Cauchy's theorem,

$$
\int_{0}^{1} f(x) d x=\frac{1}{2} \int_{\mathcal{C}_{1}} f(x) d x-\frac{1}{2} \int_{\mathcal{C}_{2}} f(x) d x .
$$


Again, by Cauchy's residue theorem,

$$
\int_{\mathcal{C}_{1}+\mathcal{C}_{2}} f(z) \cot \left(\pi\left(n z-t_{\nu}\right)\right) d z= \begin{cases}\frac{2 i}{n} \sum_{k=0}^{n-1} f\left(\left(k+t_{\nu}\right) / n\right), & -1<\nu<1 \\ \frac{2 i}{n} \sum_{k=0}^{n}{ }^{\prime \prime} f(k / n), & \nu=1\end{cases}
$$

From equations (71), (73), (76) and (77) we have

$$
\begin{aligned}
E_{n}^{[\nu]} f= & \frac{1}{2} \int_{\mathcal{C}_{1}} f(z)\left(1+i \cot \left(\pi\left(n z-t_{\nu}\right)\right)\right) d z \\
& -\frac{1}{2} \int_{\mathcal{C}_{2}} f(z)\left(1-i \cot \left(\pi\left(n z-t_{\nu}\right)\right)\right) d z \\
= & \int_{\mathcal{C}_{2}} \frac{f(z) d z}{\left\{\exp \left[-2 \pi i\left(n z-t_{\nu}\right)\right]-1\right\}} \\
& -\int_{\mathcal{C}_{1}} \frac{f(z) d z}{\left\{\exp \left[2 \pi i\left(n z-t_{\nu}\right)\right]-1\right\}} .
\end{aligned}
$$

From condition (ii) we see that the contributions to the integrals over $\mathcal{C}_{1}$ and $\mathcal{C}_{2}$ from $B C$ and $F G$ respectively tend to zero as $Y \rightarrow \infty$. If we let $E_{n, 0}^{[\nu]} f$ denote the contribution to $E_{n}^{[\nu]} f$ from the integrals over $A B$ and $G H$ then we find that

$$
E_{n, 0}^{[\nu]} f=2 \Im\left\{\int_{0}^{Y} \frac{f(i y) d y}{\exp \left[2 \pi\left(n y+i t_{\nu}\right)\right]-1}\right\}
$$


Similarly, if $E_{n, 1}^{[\nu]} f$ denotes the contribution to $E_{n}^{[\nu]} f$ from integrals over $C D$ and EF then

$$
E_{n, 1}^{[\nu]} f=-2 \Im\left\{\int_{0}^{Y} \frac{f(1+i y) d y}{\exp \left[2 \pi\left(n y+i t_{\nu}\right)\right]-1}\right\} .
$$

From equations (79) and (80) and letting $Y \rightarrow \infty$, equation (75) follows.

Suppose now that $\gamma_{r}$ denotes a sigmoidal transformation of order $r>1$. From equation (72), on writing $t=\gamma_{r}(\tau)$, we have

$$
I f=\int_{0}^{1} f\left(\gamma_{r}(\tau)\right) \gamma_{r}^{\prime}(\tau) d \tau .
$$

Applying the offset trapezoidal rule to the integrand $f\left(\gamma_{r}(\tau)\right) \gamma_{r}^{\prime}(\tau)$ gives a quadrature $\operatorname{sum} Q_{n}^{[\nu, r]} f$, say, where

$$
Q_{n}^{[\nu, r]} f:= \begin{cases}\frac{1}{n} \sum_{j=0}^{n-1} f\left(\gamma_{r}\left(\left(j+t_{\nu}\right) / n\right)\right) \gamma_{r}^{\prime}\left(\left(j+t_{\nu}\right) / n\right), & -1<\nu<1, \\ \frac{1}{n} \sum_{j=1}^{n-1} f\left(\gamma_{r}(j / n)\right) \gamma_{r}^{\prime}(j / n), & \nu=1 .\end{cases}
$$

We recall that for $\nu=1$, the integrand vanishes when $j=0$ and $n$ since $r>1$ and $\gamma_{r}^{\prime}(0)=\gamma_{r}^{\prime}(1)=0$. We define the truncation error $E_{n}^{[\nu, r]} f$ by

$$
E_{n}^{[\nu, r]} f:=I f-Q_{n}^{[\nu, r]} f .
$$

Let us define

$$
g_{r}(\tau):=f\left(\gamma_{r}(\tau)\right) \gamma_{r}^{\prime}(\tau), \quad 0 \leq \tau \leq 1
$$


We need to extend the definition of $g_{r}$ from the interval $[0,1]$ into the strip $S$. For the function $\gamma_{r}$ in $S$ we shall, in particular, replace condition (ii) of Definition 1.1(a) by

$$
\gamma_{r}(z)+\gamma_{r}(1-z)=1 \quad \forall z \in S .
$$

From this we see that

$$
\gamma_{r}(1+i y)=1-\gamma_{r}(-i y), \quad 0 \leq y<\infty,
$$

and

$$
\gamma_{r}^{\prime}(1+i y)=\gamma_{r}^{\prime}(-i y), \quad 0 \leq y<\infty .
$$

With these comments we now have the following theorem.

Theorem 4.2 Suppose that the definition of $g_{r}$ can be continued into the strip $S$ such that

(i) $g_{r}$ is continuous in $S$ and holomorphic in $\operatorname{int}(S)$;

(ii) $g_{r}(z)=o(\exp (2 \pi n|\Im z|))$ as $\Im z \rightarrow \pm \infty$ in $S$, uniformly with respect to $\Re z$.

Then

$$
\begin{aligned}
& E_{n}^{[\nu, r]} f=E_{n}^{[\nu]} g_{r}= \\
& -2 \Im\left\{\int_{0}^{\infty} \frac{f\left(1-\gamma_{r}(-i y)\right) \gamma_{r}^{\prime}(-i y)-f\left(\gamma_{r}(i y)\right) \gamma_{r}^{\prime}(i y)}{\exp \left[2 \pi\left(n y+i t_{\nu}\right)-1\right]} d y\right\} .
\end{aligned}
$$


Proof. It is obvious that $E_{n}^{[\nu, r]} f=E_{n}^{[v]} g_{r}$ and equation (88) follows immediately on applying the Abel-Plana formula, Theorem 4.1, to $g_{r}$ and using equations (86) and (87).

Equation (88) will be taken as the starting point for the asymptotic estimates of $E_{n}^{[\nu, r]} f$ for $n \gg 1$, to be discussed in the next two subsections.

\subsection{Asymptotic estimates for $E_{n}^{[\nu, r]} f$ when $f$ is holomorphic on $S$}

In making our asymptotic estimates our basic assumption is that $n$ is chosen large enough so that the major contribution to the integral in (88) comes from the neighbourhood of $y=0$.

Theorem 4.3 In addition to the conditions of Theorem 4.2 let us assume that $f$ is holomorphic at both 0 and 1 . Then, for large enough $n$,

$$
\begin{aligned}
E_{n}^{[\nu, r]} f \sim & \sum_{k=0}^{\infty} \frac{\left((-1)^{k} f^{(k)}(1)+f^{(k)}(0)\right)}{k !} \times \\
& \times \int_{0}^{\infty} \frac{\left[\cos \left(2 \pi t_{\nu}\right)-\exp (-2 \pi n y)\right] \Im\left[\gamma_{r}^{k}(i y) \gamma_{r}^{\prime}(i y)\right]}{\cosh (2 \pi n y)-\cos \left(2 \pi t_{v}\right)} d y+ \\
& +\sin \left(2 \pi t_{\nu}\right) \sum_{k=0}^{\infty} \frac{\left((-1)^{k} f^{(k)}(1)-f^{(k)}(0)\right)}{k !} \times
\end{aligned}
$$




$$
\times \int_{0}^{\infty} \frac{\Re\left[\gamma_{r}^{k}(i y) \gamma_{r}^{\prime}(i y)\right]}{\cosh (2 \pi n y)-\cos \left(2 \pi t_{\nu}\right)} d y .
$$

Proof. Recall that we are assuming that both $f$ and $\gamma_{r}$ are real on $[0,1]$. Since we are assuming that $f$ is holomorphic at both 0 and 1 then, in some region of 0 and 1 we have respectively, that

$$
f\left(\gamma_{r}(i y)\right)=\sum_{k=0}^{\infty} \frac{f^{(k)}(0)}{k !} \gamma_{r}^{k}(i y),
$$

and

$$
f\left(1-\gamma_{r}(-i y)\right)=\sum_{k=0}^{\infty} \frac{(-1)^{k} f^{(k)}(1)}{k !} \gamma_{r}^{k}(-i y) .
$$

If we formally substitute (90) and (91) into (88) and assume that the interchange of summation and integration is permissible, we obtain (89) after some tedious algebra.

Note that in (89) we have used the symbol " $\sim$ " to denote "asymptotically equal to" i.e. $a(n) \sim b(n)$ implies that $a(n) / b(n) \rightarrow 1$ in the limit as $n \rightarrow \infty$.

Let us assume that near $x=0$ we can write

$$
\gamma_{r}(x)=c_{0}(r) x^{r}\left\{1+\sum_{k=1}^{\infty} d_{k}(r) x^{k}\right\},
$$


where $c_{0}(r)$ and $d_{k}(r)$, for all $k \in \mathbf{N}$, are real. From equation (92) we can write

$$
\gamma_{r}\left(e^{i \pi / 2} y\right)=e^{i r \pi / 2} \delta_{r}(y)+e^{i(r-1) \pi / 2} \epsilon_{r}(y)
$$

say, where the real functions $\delta_{r}$ and $\epsilon_{r}$ are defined by

$$
\left\{\begin{array}{c}
\delta_{r}(y):=c_{0}(r) y^{r}\left\{1+\sum_{k=1}^{\infty}(-1)^{k} d_{2 k}(r) y^{2 k}\right\}, \\
\epsilon_{r}(y):=c_{0}(r) y^{r-1} \sum_{k=1}^{\infty}(-1)^{k} d_{2 k-1}(r) y^{2 k} .
\end{array}\right.
$$

Again, from equation (93),

$$
\gamma_{r}^{\prime}\left(e^{i \pi / 2} y\right)=e^{i(r-1) \pi / 2} \delta_{r}^{\prime}(y)-e^{i r \pi / 2} \epsilon_{r}^{\prime}(y) .
$$

With these preliminaries established we now have the following theorem.

Theorem 4.4 Let $f$ satisfy the conditions of Theorem 4.3. For any sigmoidal transformation $\gamma_{r}$ of order $r>1$ and for $-1<\nu \leq 1$ then for $n \gg 1$

$$
\begin{aligned}
n^{r} E_{n}^{[\nu, r]} f \sim & -r c_{0}(r)\left\{f(0)\left[\zeta\left(1-r, t_{\nu}\right)+(1+1 / r) d_{1}(r) \zeta\left(-r, t_{\nu}\right) / n\right]+\right. \\
& \left.+f(1)\left[\zeta\left(1-r, 1-t_{\nu}\right)+(1+1 / r) d_{1}(r) \zeta\left(-r, 1-t_{\nu}\right) / n\right]\right\}+ \\
& +O\left(1 / n^{\min (2, r)}\right),
\end{aligned}
$$

where $\zeta(\cdot, \cdot)$ denotes the generalised Riemann zeta function. 
Proof. In (89) we take the first term in each sum and write

$$
\left\{\begin{array}{c}
\gamma_{r}(i y)=c_{0}(r) e^{i \pi r / 2} y^{r}+c_{0}(r) d_{1}(r) e^{i(r+1) \pi / 2} y^{r+1}+O\left(y^{r+2}\right), \\
\gamma_{r}^{\prime}(i y)=r c_{0}(r) e^{i(r-1) \pi / 2} y^{r-1}+(r+1) c_{0}(r) d_{1}(r) e^{i r \pi / 2} y^{r}+O\left(y^{r+1}\right) .
\end{array}\right.
$$

We shall make use of the integrals

$$
\int_{0}^{\infty} \frac{y^{r-1} d y}{\cosh (2 \pi n y)-\cos \left(2 \pi t_{\nu}\right)}=\frac{2 \Gamma(r)}{(2 \pi n)^{r}} \sum_{s=1}^{\infty} \frac{1}{s^{r}} \cdot \frac{\sin \left(2 \pi s t_{\nu}\right)}{\sin \left(2 \pi t_{\nu}\right)}
$$

and

$$
\int_{0}^{\infty} \frac{y^{r-1}\left[\cos \left(2 \pi t_{\nu}\right)-\exp (-2 \pi n y)\right] d y}{\cosh (2 \pi n y)-\cos \left(2 \pi t_{\nu}\right)}=\frac{2 \Gamma(r)}{(2 \pi n)^{r}} \sum_{s=1}^{\infty} \frac{\cos \left(2 \pi s t_{\nu}\right)}{s^{r}}
$$

see, for example, Erdélyi et al [3, §6.6(5) and (8)]. In addition, we need the following representation of the generalised Riemann zeta function

$$
\zeta(1-r, q)=\frac{2 \Gamma(r)}{(2 \pi)^{r}} \sum_{s=1}^{\infty} \frac{\cos (2 \pi s q-r \pi / 2)}{s^{r}},
$$

valid for $r>1$ and $0 \leq q \leq 1$. (See, for example, Gradshteyn and Ryzhik [4, $\S 9.521(2)]$. After some straightforward algebra, we obtain the terms in $f(0)$ and $f(1)$ in equation (96).

For the term $O\left(1 / n^{\min (2, r)}\right)$ we note that in both (98) and (99) the integral of the term involving $y^{r-1}$ is $O\left(1 / n^{r}\right)$. Thus if in the terms involving $f(0)$ and 
$f(1)$ we had kept higher order terms in $\gamma_{r}^{\prime}\left(e^{i \pi / 2} y\right)$ this would have given terms of order $1 / n^{r+2}$. Again, if we had used the terms in (89) involving $f^{\prime}(0)$ and $f^{\prime}(1)$ we would obtain terms of order $1 / n^{2 r}$. Since we have assumed $r>1$, (96) gives the dominant terms in the asymptotic expansion of $n^{r} E_{n}^{[\nu, r]} f$. This completes the proof.

There are two special cases which are worth noting. The first, in which $\nu=0$ gives $t_{0}=1 / 2$ which corresponds to the "mid-point" rule and the second, where $\nu=1$ so that $t_{\nu}=1$, corresponds to the "trapezoidal" rule.

Corollary 4.5 Under the conditions of Theorem 4.4

$$
\begin{aligned}
(2 \pi n)^{r} E_{n}^{[0, r]} f \sim & 2 c_{0}(r) \Gamma(r+1)(f(0)+f(1)) \times \\
& \times\left\{\cos (r \pi / 2)\left(1-2^{1-r}\right) \zeta(r)-\right. \\
& \left.-\sin (r \pi / 2)(r+1) d_{1}(r)\left(1-2^{-r}\right) \zeta(r+1) /(2 \pi n)\right\} \\
& +O\left(1 / n^{\min (2, r)}\right)
\end{aligned}
$$

and

$$
\begin{aligned}
(2 \pi n)^{r} E_{n}^{[1, r]} f \sim & 2 c_{0}(r) \Gamma(r+1)(f(0)+f(1)) \cos (r \pi / 2) \zeta(r)- \\
& \left.-\sin (r \pi / 2)(r+1) d_{1}(r) \zeta(r+1) /(2 \pi n)\right\} \\
& +O\left(1 / n^{\min (2, r)}\right)
\end{aligned}
$$


Proof. This follows immediately from (96) on using the definition of the Riemann zeta function; see Gradshteyn and Ryzhik [4, $99.522(1)]$.

From equations (101) and (102) it can be seen that if $d_{1}(r)=0$ and $r$ is an odd integer then the terms in \{\} vanish and we need to consider higher order terms. On the other hand with $d_{1}(r)=0$ and $r$ an even integer then the terms in \{\} will not be zero and the error behaves like $O\left(1 / n^{r}\right)$ which is unremarkable.

In the more general case given by (96) we see that for all $t_{\nu} \in(0,1]$ if $d_{1}(r)=$ 0 then when $r$ is an even integer

$$
(2 \pi n)^{r} E_{n}^{[\nu, r]} f \sim 2 \Gamma(r)(-1)^{1+r / 2}(f(0)+f(1)) \sum_{s=1}^{\infty} \frac{\cos \left(2 \pi s t_{\nu}\right)}{s^{r}}+O\left(1 / n^{2}\right)
$$

and, when $r$ is an odd integer $\geq 3$,

$$
(2 \pi n)^{r} E_{n}^{[\nu, r]} f \sim 2 \Gamma(r)(-1)^{(r-1) / 2}(f(0)-f(1)) \sum_{s=1}^{\infty} \frac{\sin \left(2 \pi s t_{\nu}\right)}{s^{r}}+O\left(1 / n^{2}\right) .
$$

We shall now suppose that $r$ is an odd integer $\geq 3$ and that for all $k \in \mathbf{N}$, $d_{2 k-1}(r)=0$ so that $\varepsilon_{r}(y)=0$; see (92) and (93). As the next theorem shows we have an improved rate of convergence.

Theorem 4.6 Suppose that, in addition to the condition of Theorem 4.3, $r=$ $2 m+1, m \in \mathbf{N}$, and $\gamma_{2 m+1}$ is such that $\varepsilon_{2 m+1}$ is zero. If any of the following 
conditions is satisfied: (A) $t_{\nu}=1 / 2,(B) t_{\nu}=1,(C) f(1)=f(0)$,then

$$
\begin{aligned}
(2 \pi n)^{2(2 m+1)} E_{n}^{[\nu, 2 m+1]} f \sim & \left(c_{0}(2 m+1)\right)^{2} \Gamma(4 m+3)\left(f^{\prime}(1)-f^{\prime}(0)\right) \times \\
& \times\left(\sum_{s=1}^{\infty} \frac{\cos \left(2 \pi s t_{v}\right)}{s^{4 m+2}}\right)\left(1+O\left(\frac{1}{n^{2}}\right)\right) \\
& +O\left(\frac{1}{n^{2 m+1}}\right) .
\end{aligned}
$$

Proof. Since $r=2 m+1$ and $\varepsilon_{2 m+1}=0$ we have

$$
\gamma_{2 m+1}\left(e^{i \pi / 2} y\right)=(-1)^{m} e^{i \pi / 2} \delta_{2 m+1}(y)
$$

and

$$
\gamma_{2 m+1}^{\prime}\left(e^{i \pi / 2} y\right)=(-1)^{m} \delta_{2 m+1}^{\prime}(y)
$$

Consequently

$$
\Re\left[\gamma_{r}^{k}(i y) \gamma_{r}^{\prime}(i y)\right]=(-1)^{m(k+1)} \cos (k \pi / 2) \delta_{2 m+1}^{k}(y) \delta_{2 m+1}^{\prime}(y)
$$

and

$$
\Im\left[\gamma_{r}^{k}(i y) \gamma_{r}^{\prime}(i y)\right]=(-1)^{m(k+1)} \sin (k \pi / 2) \delta_{2 m+1}^{k}(y) \delta_{2 m+1}^{\prime}(y) .
$$

Substituting these results into (89) gives

$$
E_{n}^{[\nu, 2 m+1]} f \sim \sum_{k=1}^{\infty} \frac{(-1)^{k}\left(f^{(2 k-1)}(1)-f^{(2 k-1)}(0)\right)}{(2 k-1) !} \times
$$




$$
\begin{aligned}
& \times \int_{0}^{\infty} \frac{\left[\cos \left(2 \pi t_{\nu}\right)-\exp (-2 \pi n y)\right] \delta_{2 m+1}^{2 k-1}(y) \delta_{2 m+1}^{\prime}(y) d y}{\cosh (2 \pi n y)-\cos \left(2 \pi t_{\nu}\right)} \\
& +(-1)^{m} \sin \left(2 \pi t_{\nu}\right) \sum_{k=0}^{\infty} \frac{(-1)^{k}\left(f^{(2 k)}(1)-f^{(2 k)}(0)\right)}{(2 k) !} \times \\
& \times \int_{0}^{\infty} \frac{\delta_{2 m+1}^{2 k}(y) \delta_{2 m+1}^{\prime}(y) d y}{\cosh (2 \pi n y)-\cos \left(2 \pi t_{\nu}\right)} .
\end{aligned}
$$

Now near $y=0$ we have

$$
\delta_{2 m+1}(y)=c_{0}(2 m+1) y^{2 m+1}\left(1+O\left(y^{2}\right)\right)
$$

and

$$
\delta_{2 m+1}^{\prime}(y)=(2 m+1) c_{0}(2 m+1) y^{2 m}\left(1+O\left(y^{2}\right)\right)
$$

we find that

$$
\delta_{2 m+1}^{2 k-1}(y) \delta_{2 m+1}^{\prime}(y)=\left(c_{0}(2 m+1)\right)^{2 k}(2 m+1) y^{2 k(2 m+1)-1}\left(1+O\left(y^{2}\right)\right)
$$

From (99) and (107) we have

$$
\begin{aligned}
& \int_{0}^{\infty} \frac{\left[\cos \left(2 \pi t_{\nu}\right)-\exp (-2 \pi n y)\right] \delta_{2 m+1}^{2 k-1}(y) \delta_{2 m+1}^{\prime}(y) d y}{\cosh (2 \pi n y)-\cos \left(2 \pi t_{\nu}\right)} \\
= & (2 m+1)\left(c_{0}(2 m+1)\right)^{2 k} \frac{2 \Gamma(2 k(2 m+1))}{(2 \pi n)^{2 k(2 m+1)}} \\
& \times \sum_{s=1}^{\infty} \frac{\cos \left(2 \pi s t_{\nu}\right)}{s^{2 k(2 m+1)}}\left(1+O\left(1 / n^{2}\right)\right) .
\end{aligned}
$$


Again, from (98) and (107) we find

$$
\begin{aligned}
& \int_{0}^{\infty} \frac{\delta_{2 m+1}^{2 k}(y) \delta_{2 m+1}^{\prime}(y) d y}{\cosh (2 \pi n y)-\cos \left(2 \pi t_{\nu}\right)} \\
= & (2 m+1)\left(c_{0}(2 m+1)\right)^{2 k+1} \times \frac{2 \Gamma((2 k+1)(2 m+1))}{(2 \pi n)^{(2 m+1)(2 k+1)}} \\
& \times \sum_{s=1}^{\infty} \frac{1}{s^{(2 k+1)(2 m+1)}} \cdot \frac{\sin \left(2 \pi s t_{\nu}\right)}{\sin \left(2 \pi t_{\nu}\right)}\left(1+O\left(1 / n^{2}\right)\right) .
\end{aligned}
$$

By substituting (110) and (111) into (108) and taking the first non-vanishing term in each sum we see that (105) follows for each of conditions (A), (B) and (C). The details are omitted.

The result (105) is particularly good, for if we compare it with (96) we see that under the conditions of Theorem 4.6, $E_{n}^{[\nu, r]} f \sim O\left(1 / n^{2 r}\right)$ rather than $O\left(1 / n^{r}\right)$ of Theorem 4.4. This dramatic improvement in convergence was first observed by Sidi [15] for a whole class of sigmoidal transformations of which Transformation 3.4 is an example

Let us consider condition (C) of Theorem 4.6. If $f(1) \neq f(0)$ then if we define $F(x):=f(x)+(f(0)-f(1)) x$ we see that $F(1)=F(0)(=f(0))$. Furthermore,

$$
\int_{0}^{1} f(x) d x=\int_{0}^{1} F(x) d x-\frac{1}{2}(f(0)-f(1))
$$

and we could apply Theorem 4.6 to the function $F$. 
In the next subsection we consider asymptotic estimates of the error when $f$, instead of being holomorphic at 0 and 1 , has algebraic singularities at these points.

\subsection{Asymptotic estimates for $E_{n}^{[\nu, r]} f$ when $f$ has algebraic sin- gularities at the end-points}

The case when $f$ has an algebraic singularity at either or both end-points arises frequently enough to justify its analysis. The principal result is given in Theorem 4.7 and from it we shall see that there is no special case such as that given by Theorem 4.6 in the case when $f$ is holomorphic at both end points. Before stating the theorem we note from equation (70) that we have

$$
1-t_{\nu}=t_{-\nu} \text { for }-1<\nu \leq 1 \text {. }
$$

Theorem 4.7 Suppose $f$ is defined on $S$ by

$$
f(z)=z^{\alpha}(1-z)^{\beta} g(z) \text { for } \alpha, \beta>-1,
$$

where $g$ is holomorphic on $S$, real on $[0,1]$ and such that $g(0) \neq 0, g(1) \neq 0$. Let $\gamma_{r}$ be a sigmoidal transformation of order $r, r>1$. Then for $n \gg 1$

$$
n^{r} E_{n}^{[\nu, r]} f \sim J_{\nu}(\alpha, r, n) g(0)+J_{-\nu}(\beta, r, n) g(1)
$$

where

$$
\begin{aligned}
J_{\nu}(\alpha, r, n):= & -r\left(c_{0}(r)\right)^{1+\alpha}\left\{\zeta\left(1-r(\alpha+1), t_{\nu}\right)+\right. \\
& \left.+(\alpha+1+1 / r) d_{1}(r) \zeta\left(-r(\alpha+1), t_{\nu}\right) / n\right\} / n^{\alpha r} .
\end{aligned}
$$


Proof. We proceed as we have done in the proofs of Theorems 4.4 and 4.6, starting with equation (88) and replacing $\gamma_{r}(x)$ by $c_{0}(r) x^{r}\left(1+d_{1}(r) x\right)$ and similarly for $\gamma_{r}^{\prime}(x)$. In particular we find near 1 that

$$
\begin{aligned}
& f\left(1-\gamma_{r}\left(e^{-i \pi / 2} y\right)\right) \gamma_{r}^{\prime}\left(e^{-i \pi / 2} y\right) \\
= & r\left(c_{0}(r)\right)^{1+\beta} g(1)\left\{\exp (-i \pi(\beta r+r-1) / 2) y^{\beta r+r-1}+\right. \\
& +d_{1}(r)(\beta+1+1 / r) \exp (-i \pi(\beta r+r) / 2) y^{\beta r+r}+ \\
& \left.+O\left(y^{\min (\beta r+r+1, \beta r+2 r-1)}\right)\right\} .
\end{aligned}
$$

Again we make use of equations (98)-(100) so that after some tedious algebra we obtain equations (114) and (115).

Suppose in Theorem 4.7 we have a transformation $\gamma_{r}$ for which $d_{1}(r)=0$ and also that $\nu=1$, or $t_{\nu}=1$. Then a straightforward calculation shows that the right hand side of (114) will be zero if we can choose $r$ such that both $r(1+\alpha)$ and $r(1+\beta)$ are odd integers. We would then have to consider the next term in the expansion. However, we shall not pursue this matter here. Suffice it to say that there is no dramatic improvement in the rate of convergence by choosing $r$ to be an odd integer as happened in the previous case which essentially corresponded to having $\alpha=\beta=0$.

We complete this section by considering the asymptotic estimate of the error $E_{n}^{[1, \infty]} f$ when the sigmoidal transformation is chosen to be of infinite order; in particular we shall consider the IMT transformation [5], see Transformation 3.3. We have the following result 
Theorem 4.8 Suppose $f$ is defined as in Theorem 4.7. Let $\gamma$ be the IMT transformation defined by equations (32), (34), (35) and (38). Then for $n \gg 1$ and $\nu=1$

$$
E_{n}^{[1, \infty]} f=g(0) J(\alpha, n)+g(1) J(\beta, n)
$$

where

$$
\begin{aligned}
J(\alpha, n) \sim & \frac{-2 \sqrt{\pi}(1+\alpha)^{\alpha+1 / 4}}{(Q e)^{1+\alpha}(2 \pi n)^{\alpha+3 / 4}} \exp (-\sqrt{4 \pi n(1+\alpha)}) \times \\
& \times \cos (\sqrt{4 \pi n(1+\alpha)}+3 \pi / 8+\pi \alpha / 2),
\end{aligned}
$$

$$
Q=\int_{0}^{1} \exp (-1 / x-1 /(1-x)) d x=0.00702985840660965624,
$$

correct to $20 D$.

Proof. From equation (88), on assuming that the contribution to each integral comes from the neighbourhood of zero, which is reasonable since $n \gg 1$, we find

$$
\begin{aligned}
E_{n}^{[1, \infty]} f \sim & 2 g(1) \int_{0}^{\infty} \frac{\Im\left[(\gamma(-i y))^{\beta} \gamma^{\prime}(-i y)\right]}{\exp (2 \pi n y)-1} d y \\
& -2 g(0) \int_{0}^{\infty} \frac{\Im\left[(\gamma(i y))^{\alpha} \gamma^{\prime}(i y)\right]}{\exp (2 \pi n y)-1} d y
\end{aligned}
$$


Now for $|x| \ll 1$ we have, on integration once by parts,

$$
\gamma(x)=x^{2} e^{-1 / x}(1+O(|x|)) / Q e
$$

and

$$
\gamma^{\prime}(x)=e^{-1 / x}(1+O(|x|)) / Q e .
$$

On neglecting the terms in $O(|x|)$, replacing $x$ by $e^{i \pi / 2} y$ we find from equation (119) that $E_{n}^{[1, \infty]} f$ is of the form given by equation (116) where

$$
J(\alpha, n)=\frac{2}{(Q e)^{1+\alpha}} \int_{0}^{\infty} \frac{y^{2 \alpha} \sin (\pi \alpha+(1+\alpha) / y)}{\exp (2 \pi n y)-1} d y .
$$

In order to estimate $J(\alpha, n)$ for $n \gg 1$ we define the complex integral $\mathcal{J}(\alpha, n)$ by

$$
\mathcal{J}(\alpha, n)=\int_{\mathcal{C}} e^{-2 \pi n z} z^{2 \alpha} \exp [i(\pi \alpha+(1+\alpha) / z)] d z
$$

where $\mathcal{C}$ is a suitably chosen contour from 0 to $\infty$. Then we shall suppose that

$$
J(\alpha, n) \sim 2 \Im \mathcal{J}(\alpha, n) /(Q e)^{1+\alpha} .
$$

We obtain the value of $\mathcal{J}(\alpha, n)$ by the method of steepest descents. If we write

$$
\mathcal{J}(\alpha, n)=\int_{\mathcal{C}} z^{2 \alpha} \exp \psi(z) d z
$$

and if $\zeta$ is such that $\psi^{\prime}(\zeta)=0, \zeta$ being the only zero of $\psi^{\prime}$ on $\mathcal{C}$, then the method of steepest descents gives

$$
\mathcal{J}(\alpha, n) \sim \sqrt{2 \pi} \zeta^{2 \alpha} \exp \left[i\left(\pi-\arg \psi^{\prime \prime}(\zeta)\right) / 2\right] \exp [\psi(\zeta)] /\left|\psi^{\prime \prime}(\zeta)\right|^{1 / 2}
$$


We find that

$$
\begin{aligned}
\zeta & =((1+\alpha) / 2 \pi n)^{1 / 2} \exp (-i \pi / 4) \\
\left|\psi^{\prime \prime}(\zeta)\right| & =2(2 \pi n)^{3 / 2}(1+\alpha)^{-1 / 2} \\
\arg \psi^{\prime \prime}(\zeta) & =5 \pi / 4 \\
\exp [\psi(\zeta)] & =\exp \left[-(4 \pi n(1+\alpha))^{1 / 2}\right] \exp \left[i\left(\pi \alpha+(4 \pi n(1+\alpha))^{1 / 2}\right)\right] .
\end{aligned}
$$

The substitution of these values into equation (123) and the use of equation (122) recovers for us equation (118) and the theorem is proved.

This result agrees with that given by Iri, Moriguti and Takasawa [5] so we shall not discuss this transformation any further here. Suffice it to say that they consider quite a few numerical examples which demonstrate the accuracy of this result. However, one does question whether the function $f_{0.5}(x)$ of [5, equation, (4.1)] should be $\sqrt{x} \sqrt{1-x} / 2$ and $\operatorname{not}(\sqrt{x}+\sqrt{1-x}) / 2$.

We shall conclude this section by stating, without proof, a generalization of Theorem 4.8.

Theorem 4.9 Under the same conditions as in Theorem 4.8,

$$
E_{n}^{[\nu, \infty]} f=g(0) J_{\nu}(\alpha, n)+g(1) J_{\nu}(\beta, n)
$$

where for $n \gg 1$ and $-1<\nu \leq 1$

$$
J_{\nu}(\alpha, n) \sim \frac{-2 \sqrt{\pi}(1+\alpha)^{\alpha+1 / 4}}{(Q e)^{1+\alpha}(2 \pi n)^{\alpha+3 / 4}} \exp (-\sqrt{4 \pi n(1+\alpha)}) \times
$$




$$
\times \cos \left(\sqrt{4 \pi n(1+\alpha)}-2 \pi t_{\nu}+(3+4 \alpha) \pi / 8\right) .
$$

The proof is similar to that given for Theorem 4.8 and it is obvious that when $\nu=1$, so that $t_{\nu}=1$, the result of Theorem 4.8 is recovered.

\section{Some numerical results}

Before giving some explicit numerical results let us consider the special case of a sigmoidal transformation of order $2 m+1$ which satisfies equation (106). This, in particular, implies that

$$
\gamma_{2 m+1}\left(e^{i \pi / 2} y\right)+\gamma_{2 m+1}\left(e^{-i \pi / 2} y\right)=0, \quad y \in \mathbf{R}^{+}
$$

that is, $\gamma_{2 m+1}\left(e^{i \pi / 2} y\right)$ is purely imaginary. We recall that Sidi's Transformation 3.4 was obtained by assuming that $f$ satisfies equation (32) with the function $h$ satisfying equation (33), so that

$$
\gamma(x)=\frac{1}{Q} \int_{0}^{x} h(\xi) d \xi .
$$

We ask what additional condition equation (125) imposes on $h$. With $\gamma$ defined by equation (126), equation (125) implies that

$$
\int_{0}^{e^{i \pi / 2} y} h(\xi) d \xi+\int_{0}^{e^{-i \pi / 2} y} h(\xi) d \xi=0 .
$$


On putting $\xi=-\eta$ in the second integral we require

$$
\int_{0}^{e^{i \pi / 2} y}(h(\xi)-h(-\xi)) d \xi=0 \quad \forall y \in \mathbf{R}^{+}
$$

which will certainly be true if we choose $h$ to be an even function. This, together with $h(\xi)=h(1-\xi)$ (see equation (33)), implies that if we choose $h$ to be even, periodic of period 1 and such that $h(\xi)=O\left(\xi^{2 m}\right)$ near $\xi=0$ then we have an appropriate sigmoidal transformation of order $(2 m+1)$ satisfying equation (106). We recall that Sidi chose $h(x)=\sin ^{2 m}(\pi x)$, which satisfies all these conditions.

We can argue in a similar way when $f$ is defined by equation (58) so that $\gamma$ is defined by equation (61) provided $h$ satisfies equation (59). To satisfy equation (125) we find that we now require $h$ to be an odd function with period 1. Thus if, in addition, $h(\xi)=O\left(\xi^{2 m-1}\right)$ near $\xi=0$, then $\gamma$ as defined by equation (61) will be sigmoidal of order $(2 m+1)$ and satisfying equation (106). In Transformation 3.7 we chose $h(x)=\sin ^{2 m-1}(2 \pi x)$ which obviously satisfies these conditions. Writing $h(x)=\sin ^{2 m-1}(2 \pi x) \cos (2 \pi x)$ essentially recovers Sidi's Transformation 3.4.

Let us now consider some numerical examples.

Example 5.1 Consider the evaluation of $I f=\int_{0}^{1} \exp (x) d x$. We shall illustrate this by the use of only two transformations; those given by Transformations 2.1 and 3.7. We compare the actual computed errors $n^{r} E_{n}^{[1, r]} f$ with the asymptotic estimate given by equation (102) in the case of Transformation 2.1 and for Transformation 3.7 with the asymptotic estimate for $n^{2 r} E_{n}^{[1, r]} f$ as given by equation (105) 


\begin{tabular}{ccccc} 
& \multicolumn{2}{c}{$\mathrm{r}=3.0$} & \multicolumn{2}{c}{$\mathrm{r}=3.5$} \\
$n$ & $n^{3} E_{n}^{[1,3]} f^{7 / 2} E_{n}^{[1,3.5]} f$ & eqn (102) \\
\hline \hline 20 & -0.0184942 & -0.0185914 & -0.122490 & -0.123843 \\
40 & -0.00928357 & -0.00929570 & -0.117016 & -0.117341 \\
60 & -0.00619354 & -0.00619714 & -0.115031 & -0.115174 \\
80 & -0.00464634 & -0.00464785 & -0.114011 & -0.114090
\end{tabular}

Table 5.1: Errors and estimates for Transformation 2.1

We note for Transformation 2.1 that $c_{0}(r)=1$ and $d_{1}(r)=r$. This transformation allows $r$ to be a non-integer and we have illustrated this in Table 5.1. In the cases displayed we see that the asymptotic estimates obtained for $n \gg 1$ agree well with the actual errors.

\begin{tabular}{ccc}
$n$ & $n^{6} E_{n}^{[1,3]} f$ & $n^{10} E_{n}^{[1,5]} f$ \\
\hline \hline 20 & -0.897960 & -1046 \\
40 & -0.888656 & -925 \\
60 & -0.886978 & -905 \\
80 & -0.886619 & -898 \\
100 & -0.885958 & -895 \\
$\infty$ & -0.885589 & -889
\end{tabular}

Table 5.2: Errors and estimates for Transformation 3.7 
In the second column of Table 5.2 we have given the quadrature error $n^{6} E_{n}^{[1,3]} f$ for $n=20$ (20)100 in the case when we have used a third order transformation, i.e. equation (68) with $m=1$. The value corresponding to $n=\infty$ is that obtained from the estimate (105) with $m=1, \nu=1$ and $q=1$. Note that $c_{0}(3)$ is given from equation (69) with $m=1$. In the last column of Table 5.2 we have put $m=2$ and repeated the calculations. It should be noted that one needs to carry a lot of precision in this case! In both these cases the asymptotic estimate is a reasonable estimate for the actual error.

It is of interest to compare just two results from Tables 5.1 and 5.2. In each case where we have used a third order transformation we note from Table 5.1 that $E_{20}^{[1,3]} f=-2.31 \times 10^{-4}$ whereas from Table 5.2 we have $E_{20}^{[1,3]} f=-1.40 \times 10^{-8}$; the improvement is dramatic. The fifth order transformation in Table 5.2 gives $E_{20}^{[1,5]} f=-1.02 \times 10^{-10}$.

Example 5.2 Suppose that If $=\int_{0}^{1} x^{\alpha}(1-x)^{\beta} d x$ where $\alpha, \beta>-1$. We shall consider $n^{r} E_{n}^{[1, r]} f$ again for the two sigmoidal Transformations 2.1 and 3.7. In Table 5.3 we have chosen $\alpha=0.4$ and $\beta=0.9$ and have compared the actual errors with the asymptotic estimate of Theorem 4.7 for $r=3$ and $r=4$. 


\begin{tabular}{ccccc} 
& \multicolumn{2}{c}{$\mathrm{r}=3$} & \multicolumn{2}{c}{$\mathrm{r}=4$} \\
$n$ & $n^{3} E_{n}^{[1,3]} f$ & asymptotic & $n^{4} E_{n}^{[1,4]} f$ & asymptotic \\
\hline \hline 20 & $-5.309 \times 10^{-4}$ & $-5.423 \times 10^{-4}$ & $-3.250 \times 10^{-4}$ & $+1.383 \times 10^{-4}$ \\
30 & $-3.378 \times 10^{-4}$ & $-3.410 \times 10^{-4}$ & $+6.656 \times 10^{-5}$ & $+6.821 \times 10^{-5}$ \\
40 & $-2.428 \times 10^{-4}$ & $-2.441 \times 10^{-4}$ & $+4.117 \times 10^{-5}$ & $+4.174 \times 10^{-5}$ \\
50 & $-1.873 \times 10^{-4}$ & $-1.879 \times 10^{-4}$ & $+2.840 \times 10^{-5}$ & $+2.866 \times 10^{-5}$ \\
60 & $-1.512 \times 10^{-4}$ & $-1.516 \times 10^{-4}$ & $+2.100 \times 10^{-5}$ & $+2.113 \times 10^{-5}$
\end{tabular}

Table 5.3: Errors and estimates for $\alpha=0.4, \beta=0.9$ and Transformation 2.1

From Table 5.3, with the exception of the case $r=4, n=20$ where the sign of the asymptotic estimate is incorrect, we see that the agreement between the actual errors and the asymptotic errors is excellent.

In Table 5.4 we repeat the calculations for the case when $r=3$ and $r=5$ using Transformation 3.7. In this case for the asymptotic error we have that $d_{1}(r)=0$ in Theorem 4.7. 


\begin{tabular}{ccccc} 
& \multicolumn{2}{c}{ 3rd order } & \multicolumn{2}{c}{ 5th order } \\
$n$ & $n^{3} E_{n}^{[1,3]} f$ & asymptotic & $n^{5} E_{n}^{[1,5]} f$ & asymptotic \\
\hline \hline 20 & $-7.994 \times 10^{-3}$ & $-7.953 \times 10^{-3}$ & $+3.103 \times 10^{-4}$ & $+2.619 \times 10^{-4}$ \\
30 & $-4.935 \times 10^{-3}$ & $-4.924 \times 10^{-3}$ & $+4.537 \times 10^{-5}$ & $+4.224 \times 10^{-5}$ \\
40 & $-3.501 \times 10^{-3}$ & $-3.497 \times 10^{-3}$ & $+1.204 \times 10^{-5}$ & $+1.116 \times 10^{-5}$ \\
50 & $-2.681 \times 10^{-3}$ & $-2.679 \times 10^{-3}$ & $+4.389 \times 10^{-6}$ & $+4.241 \times 10^{-6}$ \\
60 & $-2.156 \times 10^{-3}$ & $-2.155 \times 10^{-3}$ & $+1.986 \times 10^{-6}$ & $+1.867 \times 10^{-6}$
\end{tabular}

Table 5.4: Errors and estimates for $\alpha=0.4, \beta=0.9$ and Transformation 3.7

Again we see that the asymptotic estimates agree well with the actual estimates for the values of $n$ given. It is of interest to compare, from Tables 5.3 and 5.4, the actual truncation error for the third order transformation for the values of $n$ given. In each case the Transformation 2.1 gives the smaller truncation error by a factor of 10. In comparing Tables 5.3 and 5.4 we see that the actual truncation errors of the 4th order Transformation 2.1 are about the same as the 5th order Transformation 3.7 for $n=10,20$ and 30. Certainly Transformation 3.7 has no dramatic advantage over Transformation 2.1 in the case when the function $f$ has algebraic singularities at the end points.

\section{Conclusion}

The effect of a sigmoidal transformation on the offset trapezoidal rule is to replace the evaluation of the integrand at equally spaced points in $[0,1]$ by the evaluation 
of a modified integrand at points which are no longer equally spaced. It is of interest to consider the ratio $\rho$ of the maximum distance between consecutive mesh points to the minimum distance. From the way in which the sigmoidal transformation is defined it would appear that for a given $n$ we could define a mesh ratio $\rho_{n}$ as

$$
\rho_{n}:=\frac{1}{n} \times \gamma_{r}^{\prime}(1 / 2) / \gamma_{r}(1 / n) .
$$

In Table 6.1 we consider $\rho_{n}$ for some of the transformations we have considered. In producing this table not only have we assumed that $n \gg 1$ but also that $r$ is an odd integer $(=2 m+1)$ and that $m \gg 1$. The calculations are routine and the details are omitted.

\begin{tabular}{cc} 
Transformation & $n^{-2 m} \rho_{n}$ \\
\hline \hline 2.1 & $2 m$ \\
2.4 with $\ell=2$ & $(2 / 3) \exp (4 / 3) / 3^{2 m}$ \\
3.3 & $2 m / \pi^{2 m}$ \\
3.5 & $4 \sqrt{\pi} m^{3 / 2} /(2 \pi)^{2 m}$
\end{tabular}

Table 6.1

In addition to the results of Table 6.1 we mention in passing that for Transformation 2.3 with $\ell=2$ (due to Sag and Szekeres [14]) we find $n e^{-n} \rho_{n} \sim 2$ so that $\rho_{n}$ increases exponentially with $n$. From Table 6.1 we see that in all cases $\rho_{n}$ grows like $n^{2 m}$, i.e. polynomial as distinct from exponential growth with $n$. This observation has relevance when we attempt to solve approximately the various 
equations, arising from boundary integral methods, where the integral is replaced with an offset trapezoidal rule after a sigmoidal transformation. Such discussion is, however, beyond the scope of this paper.

We have observed that transformations have been chosen with $\gamma_{r}^{\prime}(1 / 2)$ being independent of $r$. Kress [8] states that “... (this) property ensures, roughly speaking, that one half of the grid points is equally distributed over the total interval, whereas the other half is accumulated towards the two end points". One way of testing the distribution of points in $[0,1]$ is to define a number dist $(r, n)$ say, such that

$$
\operatorname{dist}(r, n):=\frac{2}{n}\left\{1+\sum_{k=1}^{n-1}\left|\gamma_{r}\left(\frac{k}{n}\right)-\frac{1}{2}\right|\right\} .
$$

It is readily verified that $1 / 2 \leq \operatorname{dist}(r, n) \leq 1$. Equally spaced points correspond to dist $(r, n)=1 / 2$ whereas if all the points are evenly divided between the end points 0 and 1 then $\operatorname{dist}(r, n)=1$. In Table 6.2 we exhibit dist $(r, 100)$ for $r=3$ (2) 9 and for five transformations

\begin{tabular}{cccccc} 
order & \multicolumn{5}{c}{ Transformation } \\
$r$ & 2.1 & 2.2 & 2.3 & 3.4 & 3.7 \\
\hline \hline 3 & 0.7927 & 0.7113 & 0.8077 & 0.7126 & 0.7126 \\
5 & 0.8745 & 0.7196 & 0.8794 & 0.7801 & 0.7351 \\
7 & 0.9120 & 0.7236 & 0.9141 & 0.8161 & 0.7432 \\
9 & 0.9333 & 0.7259 & 0.9343 & 0.8393 & 0.7473
\end{tabular}

Table 6.2: $\operatorname{dist}(r, 100)$ for various $r$ and transformations. 
With values of dist $(r, n)$ closer to a half being considered as "better" than those close to 1 we see that Transformation 2.2 is the "best" of these transformations with Transformation 3.7 being a close second. The "worst" transformation, perhaps not surprisingly, is Transformation 2.3.

We might conclude by asking whether a sigmoidal transformation exists having the algebraic simplicity of Transformation 2.1, with $\gamma_{r}^{\prime}(1 / 2)$ being independent of $r$ and for which we get the improved convergence rates of Transformations 3.4 and 3.7 under certain conditions.

Acknowledgement: the author thanks Professor Avram Sidi for his comments on this paper.

\section{References}

[1] R. Duduchava, D. Elliott \& W.L. Wendland, The spline collocation method for Mellin convolution equations, Universität Stuttgart, Sonderforschungsbereich 404, Bericht 96/04, 20 pp.

[2] D. Elliott \& S. Prössdorf, An algorithm for the approximate solution of integral equations of Mellin type, Numer. Math, 70, pp.427-452, 1995.

[3] A. Erdélyi, ed. Tables of Integral Transforms, vol.1, Bateman Manuscript Project. McGraw-Hill, New York, 1954. 
[4] I.S. Gradshteyn \& I.M. Ryzhik, Table of Integrals, Series and Products, 5th edition edited by A. Jeffrey, Academic Press, Boston, 1994.

[5] M. Iri, S. Moriguti \& Y. Takasawa, On a certain quadrature formula, $J$. Comput. \& Appl. Math., 17, pp.3-20, 1987.

E79, E96, E123, E126

[6] Y. Jeon, A Nyström method for boundary integral equations on domains with a piecewise smooth boundary. Integral Equations Appl. 5, pp.221-242, 1993.

[7] N.M. Korobev, Number-theoretic methods of approximate analysis, GIFL, Moscow, 1963 (Russian)

[8] R. Kress, A Nyström method for boundary integral equations in domains with corners. Numer. Math. 58, pp.145-161, 1990.

[9] D.P. Laurie, Periodizing transformations for numerical integration, J. Comput. \& Appl. Math., 66, pp. 337-344, 1996.

[10] J. Lyness, The Euler Maclaurin expansion for the Cauchy principal value integral, Numer. Math. 46, pp.611-622, 1985.

[11] M. Mori, An IMT-type double exponential formula for numerical integration, publ. of RIMS, Kyoto University, 14, pp.713-729, 1978.

E79, E81, E98, E100, E101

E108

E79, E91

[12] F.W.J. Olver, Asymptotics and Special Functions, Academic Press, New York and London, 1974. 
[13] S. Prössdorf \& A. Rathsfeld, On an integral equation of the first kind arising from a cruciform crack problem. In: V. Petkov and R. Lazarov (eds) Integral Equations and Inverse Problems, pp.210-219, Longman, Coventry, 1991.

[14] T.W. Sag \& G. Szekeres, Numerical evaluation of high-dimensional integrals. Math. Comput. 18, pp.245-253, 1964.

E79, E88, E133

[15] A. Sidi, A new variable transformation for numerical integration. In $\mathrm{Nu}-$ merical Integration IV edited by H. Brass \& G. Hämmerlin, ISNM vol.112, E79, E97, E121 pp.359-373, Birkhauser-Verlag, 1993. 